\title{
El automanejo de los pacientes con diabetes tipo 2: una revisión narrativa
}

\author{
Patients' selfmanagement of type 2 diabetes: \\ a narrative review
}

\author{
N. Campo Guinea, M.C. Portillo
}

\section{RESUMEN}

Según la Organización Mundial de la Salud para el año 2030 la cifra de personas con diabetes mellitus alcanzará valores cercanos a 366 millones en el mundo, ocupando su mayor proporción la diabetes mellitus tipo 2. Las estrategias actuales de Cronicidad y de la Diabetes presentes en España abogan por un adecuado automanejo del paciente por medio de programas y/o intervenciones apropiadas para ello. Sin embargo, existen unos conocimientos y actitudes inadecuadas que limitan la asociación paciente-profesional que las estrategias de automanejo implican. A través de esta revisión bibliográfica se pretende mostrar las necesidades reales no cubiertas que los pacientes con diabetes mellitus tipo 2 tienen a la hora de automanejar la enfermedad. Así, se asentarán las bases para el desarrollo de futuros programas efectivos que se implementen ayudando al profesional sanitario a dirigir su trabajo diario hacía su principal objetivo, la persona con diabetes mellitus tipo 2 y sus necesidades.

Palabras clave. Automanejo. Diabetes mellitus Tipo 2. Paciente. Necesidades.

\begin{abstract}
According to the World Health Organization in 2030 the number of people with diabetes mellitus will reach 366 millions in the whole world, having the diabetes mellitus type 2 the highest prevalence. The current National Chronicity and Diabetes Strategies advocate patients' selfmanagement programmes and / or interventions. Nevertheless, the lack of knowledge and inadequate attitudes hinder the associative relationship between patients and professionals that is required in these programmes. This literature review aims to show the outstanding needs that type 2 diabetes mellitus patients have when it comes to managing the illness. Thus, results from this literature review could set the bases for the development of future effective programmes, helping professionals approach patients' needs.
\end{abstract}

Key words. Selfmanagement. Type 2 Diabetes mellitus. Patient. Needs.
Universidad de Navarra. Pamplona.

Recepción: 23 de mayo de 2013

Aceptación provisional: 24 de junio de 2013

Aceptación definitiva: 15 de julio de 2013

\section{Correspondencia:}

Natalia Campo Guinea

Zaharra, 2, $1^{\mathrm{o}} \mathrm{A}$

48460 Orduña. Vizcaya

ncampog@alumni.unav.es 


\section{INTRODUCCIÓN}

La diabetes mellitus es uno de los principales problemas de salud a nivel mundial no solo por su alta prevalencia e incidencia sino también por los costes que supone, las complicaciones derivadas de la patología y las muertes que ocasiona anualmente ${ }^{1,2}$. La Organización Mundial de la Salud (OMS) estimó que en el año 2002 existían por cada 100.000 habitantes 3.000 diabéticos y ha adelantado que las previsiones para el año 2030 tornarán alrededor de 366 millones de personas en el mundo ${ }^{3}$. La mayor parte de estas personas tendrán diabetes mellitus tipo 2 (DM2) y esto es debido al envejecimiento de la población, la urbanización, la falta de actividad física y el aumento de la obesidad $^{4,5}$. Algunos autores apoyan que la etiología de esta enfermedad se debe a factores fisiológicos y de estilos de vida que podrían modificarse a través de programas educativos $^{6,7}$. Por ello, la política sanitaria de España apuesta por dar prioridad al propio automanejo y autocuidado* del individuo y la familia por medio del diseño e implantación de programas educativos con este fin. La evidencia muestra un número muy amplio de programas educativos dirigidos al automanejo del paciente y sus familias, centrándose en su mayoría en el control glicémico, el control de la medicación y los hábitos higiénico-dietéticos ${ }^{10-17}$.

A pesar de ello, en la literatura se muestra que los pacientes con DM2 tienen un inadecuado conocimiento de la naturaleza de la enfermedad, sus factores de riesgo y sus complicaciones asociadas ${ }^{18-21}$. Esto puede estar relacionado con el hecho de que las necesidades emocionales y familiares y de

* El término automanejo hace referencia a las actividades que las personas realizan para crear orden, disciplina y control de sus vidas ${ }^{8}$. En la literatura esto se aplica también al cuidado de la salud y en las enfermedades crónicas.

Por autocuidado se entiende las acciones que los individuos realizan para promover un estilo de vida saludable, para satisfacer sus necesidades sociales, emocionales y psicológicas, cuidar de un proceso crónico y prevenir más enfermedades o complicaciones ${ }^{9}$. información no están totalmente cubiertas en dichos programas ${ }^{21-23}$.

Para profundizar más en este vacío de la literatura se ha realizado una revisión bibliográfica que ayude a establecer las necesidades reales de los pacientes con DM2 con respecto al automanejo de la enfermedad que pueden servir de base para futuros programas educativos de esta índole que se implementen en el contexto español. El objetivo principal de esta revisión bibliográfica es generar conocimiento sobre los aspectos y necesidades que los pacientes con DM2 tienen para un adecuado automanejo de la enfermedad.

\section{METODOLOGÍA}

Para lograr el objetivo de este estudio, se ha realizado una revisión narrativa de la literatura en las siguientes bases de datos: CINAHL, PUBMED y COCHRANE LIBRARY.

En la figura 1 pueden verse las palabras clave empleadas y las truncaciones realizadas para incluir posibles variaciones utilizadas en la literatura.

Se han aplicado varios límites en las bases de datos, como son, publicaciones escritas en inglés o español con acceso al resumen y cuyos participantes sean humanos y adultos. Asimismo, se ha optado por ampliar el intervalo de tiempo a 10 años ya que se considera que este tema lleva varios años estudiándose en los EEUU y Reino Unido, aunque en España, sea un tema emergente. Además, se han realizado búsquedas manuales en las revistas electrónicas consideradas de mayor relevancia para el asunto a estudiar: The Diabetes Educator, Diabetes Care, Journal Of Clinical Nursing, Journal Of Advanced Nursing, Rol de Enfermería, Enfermería Clínica y Anales del Sistema Sanitario de Navarra. También, se han realizado búsquedas con la técnica bola de nieve (snowballing), revisando las listas de referencias de los artículos ya incluidos para revisión en este trabajo para verificar la existencia de artículos adicionales no emergentes en las bases de datos. La estrategia de búsqueda utilizada puede verse en la figura 2 . 


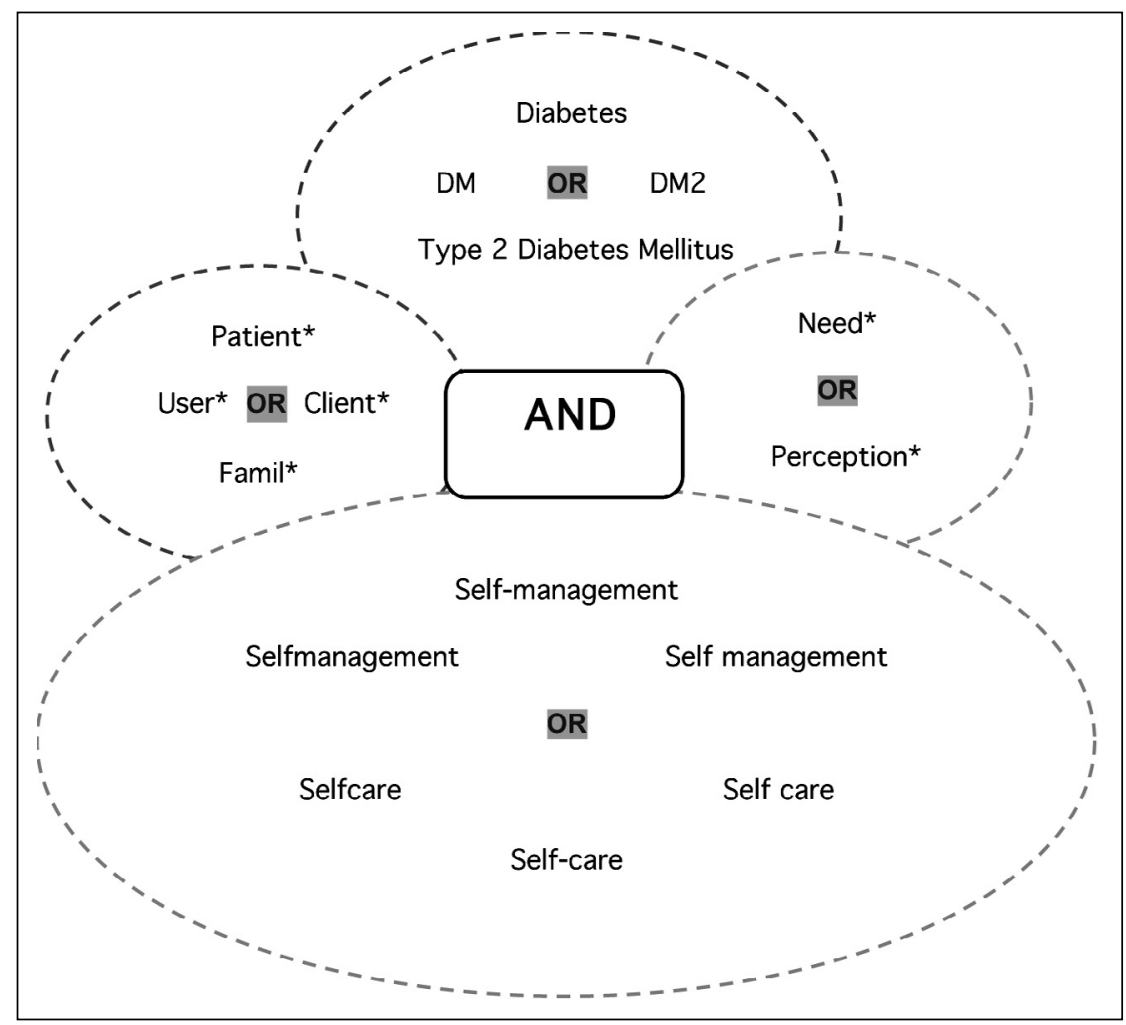

Figura 1. Estrategia llevada a cabo en las bases de datos.

Se identificaron 87 publicaciones que tras la lectura del título y el resumen eran relevantes para este trabajo. Tras leer los artículos accesibles y aplicando los criterios de inclusión y exclusión (Tabla 1), quedaron un total de 32 artículos para revisión.

Tabla 1. Criterios de inclusión y exclusión

\section{Criterios de inclusión}

Artículos que aborden necesidades, conocimientos y/o percepciones de pacientes con diabetes mellitus tipo 2 relacionados con el automanejo

\section{Criterios de exclusión}

Estudios de intervención que evalúen programas relacionados con el automanejo y que no traten ni aborden las necesidades de los pacientes con DM2

Artículos que aborden aspectos directa o indirectamente relacionados con el automanejo.

Artículos que aborden aspectos sobre el automanejo de los pacientes con diabetes mellitus tipo 2 desde otra perspectiva que no sea la de ellos

Estudios originales (cualitativos, cuantitativos, combinados y/o revisiones bibliográficas)
Estudios que incluyan a pacientes con diabetes mellitus tipo 1

\section{Literatura gris y editoriales}




\begin{tabular}{|c|c|c|c|c|c|c|c|c|c|}
\hline \multicolumn{10}{|c|}{$\begin{array}{l}1^{\circ} \text { Paso: } \\
\text { Búsqueda combinando los términos clave y los límites en las bases de datos }\end{array}$} \\
\hline \multicolumn{3}{|c|}{$\begin{array}{c}\text { Cinahl } \\
262\end{array}$} & \multicolumn{3}{|c|}{$\begin{array}{l}\text { Cochrane Library } \\
\qquad 89\end{array}$} & \multicolumn{4}{|c|}{$\begin{array}{l}\text { Pubmed } \\
\quad 906\end{array}$} \\
\hline \multicolumn{10}{|c|}{$\begin{array}{l}2^{\circ} \text { Paso: } \\
\text { Selección de artículos por título y resumen según criterios de inclusión y exclusión }\end{array}$} \\
\hline \multicolumn{3}{|c|}{ Bases de datos } & \multicolumn{7}{|c|}{ Revistas electrónicas } \\
\hline $\begin{array}{c}\text { Cinahl } \\
64\end{array}$ & $\begin{array}{c}\text { Cochrane } \\
\text { Library } \\
2\end{array}$ & $\begin{array}{l}\text { Pubmed } \\
4\end{array}$ & \multicolumn{2}{|c|}{$\begin{array}{c}\text { Journal of } \\
\text { Advanced Nursing } \\
3\end{array}$} & \multicolumn{2}{|c|}{$\begin{array}{c}\text { Journal of } \\
\text { Clinical Nursing } \\
4\end{array}$} & \multicolumn{2}{|c|}{$\begin{array}{c}\text { The Diabetes } \\
\text { Educator } \\
8\end{array}$} & $\begin{array}{c}\text { The Diabetes } \\
\text { Care } \\
2\end{array}$ \\
\hline \multicolumn{10}{|c|}{ Total: 87} \\
\hline \multicolumn{10}{|c|}{$\begin{array}{l}3^{\circ} \text { Paso: } \\
\text { Selección de publicaciones tras lectura de texto completo aplicando criterios de inclusión y exclusión }\end{array}$} \\
\hline Bola de nieve & \multicolumn{3}{|c|}{ Bases de datos } & \multicolumn{6}{|c|}{ Revistas electrónicas } \\
\hline 7 & $\begin{array}{l}\text { Cinahl } \\
19\end{array}$ & $\begin{array}{c}\text { Cochrane } \\
\text { Library } \\
0\end{array}$ & $\begin{array}{l}\text { Pubmed } \\
0\end{array}$ & & $\begin{array}{l}\text { rnal of } \\
\text { anced } \\
\text { rsing } \\
1\end{array}$ & $\begin{array}{c}\text { Journa } \\
\text { Clinic } \\
\text { Nursi } \\
2\end{array}$ & & $\begin{array}{c}\text { The } \\
\text { Diabetes } \\
\text { Educator } \\
3\end{array}$ & $\begin{array}{c}\text { The } \\
\text { Diabetes } \\
\text { Care } \\
0\end{array}$ \\
\hline \multicolumn{10}{|c|}{ Total: 32} \\
\hline \multicolumn{10}{|c|}{$\begin{array}{l}4^{\circ} \text { Paso: } \\
\text { Crítica metodológica de los } 35 \text { artículos seleccionados }\end{array}$} \\
\hline \multicolumn{4}{|c|}{26 adecuada calidad metodológica } & \multicolumn{6}{|c|}{6 no cumplen criterios de calidad } \\
\hline \multicolumn{10}{|c|}{ Total para revisión: 26} \\
\hline
\end{tabular}

Figura 2. Resultado de la estrategia de búsqueda

Una vez evaluada la calidad metodológica, siguiendo los criterios de validez metodológica establecidos por el Instituto Joanna Briggs ${ }^{24}$ un total de 26 artículos fue- ron incluidos para la revisión. En la tabla $2^{25-30}$ se reflejan los motivos por los cuáles 6 artículos fueron descartados.

Tabla 2. Artículos descartados por no cumplir criterios de calidad metodológica

\begin{tabular}{ll}
\hline \multicolumn{1}{c}{ Autor; Año } & \multicolumn{1}{c}{ Motivo principal de exclusión } \\
\hline Wellard SJ, Rennie S, King R; 2008 & No indicaciones del uso de consentimiento informado. \\
\hline Hall RF, Joseph DH, Schwartz-Barcott D; 2003 & $\begin{array}{l}\text { No indicaciones de aprobación comité ético, ni consentimiento } \\
\text { informado. }\end{array}$ \\
\hline Nunez MA, Yarandi H, Nunez-Smith M; 2011 & No indicaciones de consentimiento informado. \\
\hline Barnes L, Moss-Morris R, Kaufusi M; 2004 & No indicaciones de aprobación comité ético. \\
\hline Lin CC, Anderson RM, Hagerty BM, Lee BO; 2008 & Falta características socio-demográficas de participantes. \\
\hline $\begin{array}{l}\text { Wong M, Haswell-Elkins M, Tamwoy E, } \\
\text { McDermott R, d'Abbs P; 2005 }\end{array}$ & Falta características socio-demográficas de participantes. \\
\hline
\end{tabular}




\section{RESULTADOS}

\section{Análisis de los estudios}

De los 26 estudios incluidos en la revisión: 25 utilizaron metodología cualitativa cuyos métodos principales de recogida de datos fueron los grupos focales, entrevistas abiertas y/o observaciones y uno utilizó la metodología cuantitativa ${ }^{31}$ mediante la recogida de datos por medio de cuestionarios. Aunque esta revisión está compuesta principalmente por estudios que utilizan metodologías cualitativas no se considera una limitación en cuanto a la calidad de los resultados porque la metodología cualitativa es una opción adecuada para abordar las percepciones y experiencias de los individuos $^{32}$. Asimismo se debe tener en cuenta que la mayor parte son estudios exploratorios y descriptivos. El gran problema de estos diseños es la imposibilidad de obtener información en tiempo real, lo que dificulta la comprensión de las percepciones de los pacientes de forma integral y en un periodo de tiempo determinado. En la tabla 3 se describen los estudios incluidos y sus principales resultados.

Tabla 3. Resultados de los estudios incluidos en la revisión

\begin{tabular}{|c|c|c|c|c|}
\hline Artículo & País & Objetivo & Metodología & Resultados más relevantes \\
\hline $\begin{array}{l}\text { Abbott S, } \\
\text { Gunnell C; } \\
2004^{22}\end{array}$ & Inglaterra & $\begin{array}{l}\text { Identificar las experien- } \\
\text { cias de ancianos con DM2 } \\
\text { sobre el automanejo y la } \\
\text { sanidad }\end{array}$ & $\begin{array}{l}\text { Diseño: cualitativo explo- } \\
\text { ratorio. } \\
\text { Métodos: } 54 \text { entrevistas } \\
\text { a pacientes ancianos. La } \\
\text { mayoría británicos, sólo } \\
6 \text { de ellos eran origina- } \\
\text { rios del sur de Asia. }\end{array}$ & $\begin{array}{l}\text { Mayor demanda del profesional } \\
\text { sanitario. } \\
\text { Familia principal soporte. Falta de } \\
\text { conocimientos relacionados con el } \\
\text { porqué de las recomendaciones. } \\
\text { Demanda de continuidad en la } \\
\text { atención. }\end{array}$ \\
\hline $\begin{array}{l}\text { Adams CR; } \\
2003^{23}\end{array}$ & Inglaterra & $\begin{array}{l}\text { Describir qué significa } \\
\text { para los latinos con DM2 } \\
\text { vivir con la enfermedad }\end{array}$ & $\begin{array}{l}\text { Diseño: fenomenología } \\
\text { descriptiva. } \\
\text { Métodos: } 13 \text { entrevistas } \\
\text { abiertas en profundidad } \\
\text { a mujeres latinas de un } \\
\text { centro de salud en el sur } \\
\text { de Inglaterra. }\end{array}$ & $\begin{array}{l}\text { Importancia de la multicultura. } \\
\text { Percepción errónea sobre la etio- } \\
\text { logía de la enfermedad. Influencia } \\
\text { de la cultura. Barreras lingüísti- } \\
\text { cas en las consultas con los pro- } \\
\text { fesionales, recursos económicos } \\
\text { limitados que afecta al acceso al } \\
\text { especialista. }\end{array}$ \\
\hline
\end{tabular}

\begin{tabular}{llll}
\hline Burke JA, & Estados & Determinar la opinión de & Diseño: teoría fundamen- \\
Earley M, & Unidos & los pacientes con DM2 & tada. \\
Dixon LD, & & sobre cómo deben ser los & Métodos: 2 grupos foca- \\
Wilke A, & & encuentros con los profe- les de 4 participantes \\
Puczynski S; & & sionales para promover un cada grupo. \\
2006 $^{42}$ & & automanejo adecuado
\end{tabular}

Caban A, Estados

Walker EA, Unidos

Sanchez $\mathbf{S}$,

Mera MS;

$2008^{36}$
Examinar aspectos psi- Diseño: cualitativo. cosociales que afectan al Métodos: grupos focales automanejo de pacientes látinos que viven en EEUU con DM2 a 37 participantes. Los grupos focales se dividieron en sexos. Latinos residentes en Nueva York de atención primaria.
Perspectivas del paciente necesarias para el profesional.

Necesidad de una continuidad de cuidados. Los profesionales deben escuchar sus experiencias y miedos, dar más información efectiva.

Importancia de la multicultura.

La cultura influye en los conocimientos de los pacientes, en el automanejo e incluso en la relación con los profesionales sanitarios.

\begin{tabular}{llll}
\hline Carter- & Estados & Describir las percepciones & Diseño: cualitativo. \\
Edwards L, & Unidos & de pacientes africanas con & Métodos: grupos focales \\
Skelly AH, & & DM2 que viven en América & a 12 mujeres afro-ameri- \\
Cagle CS, & & sobre el soporte familiar y & canas hospitalizadas en \\
Appel SJ; & & los factores contextuales & una clínica del sur-oeste \\
2004 $^{41}$ & & que afectan al automanejo & de EEUU.
\end{tabular}

Importancia de la multicultura. El rol social de la mujer como multicuidador de los diferentes miembros de la familia, la falta de entendimiento familiar sobre las necesidades de las pacientes y la cultura influyen en el automanejo. 


\begin{tabular}{|c|c|c|c|c|}
\hline Artículo & País & Objetivo & Metodología & Resultados más relevantes \\
\hline $\begin{array}{l}\text { Fleming E, } \\
\text { Carter B, } \\
\text { Pettigrew J; } \\
\mathbf{2 0 0 8}^{37}\end{array}$ & Inglaterra & $\begin{array}{l}\text { Explorar la influencia de la } \\
\text { cultura musulmana Gujara- } \\
\text { ti en el automanejo en pa- } \\
\text { cientes hombres con DM2 } \\
\text { que viven en el norte de } \\
\text { Inglaterra }\end{array}$ & $\begin{array}{l}\text { Diseño: estudio de casos. } \\
\text { Métodos: observación y } \\
\text { entrevistas abiertas a } 5 \\
\text { participantes varones de } \\
\text { atención primaria. }\end{array}$ & $\begin{array}{l}\text { Importancia de la multicultura. } \\
\text { Las experiencias pasadas sobre } \\
\text { homeopatía y contexto social de } \\
\text { una cultura en relación a los con- } \\
\text { sejos familiares influyen en el auto- } \\
\text { manejo de la DM2. }\end{array}$ \\
\hline
\end{tabular}

Handley J, Nueva Zelanda Comprender las experien- Diseño: aproximación Barreras para un adecuado auto-

Gifford H;
Pullon S,

$2010^{45}$ cias vividas por pacientes con DM2 sobre el automanejo y su condición fenomenológica y teoría fundamentada.

Métodos: entrevistas semi-estructuradas a 9 participantes (5 mujeres y 4 hombres) pertenecientes a un centro de salud regional.

Describir los malentendidos que tienen los pacientes suecos con DM2 con respecto a su enfermedad, su tratamiento y su automanejo

Holmström IM, Suecia Rosenqvist U; $2005^{18}$

Hornsten A, Suecia
Lundman B,
Selstam EK,
Sandstrom H;
$\mathbf{2 0 0 5}^{50}$

Identificar las experiencias y las reflexiones de personas con DM2 sobre Suecia

Johnson M, Reino Unido
Baird W,
Goyder E;
2006 $^{\mathbf{4 8}}$

Explorar los puntos de vista y las experiencias de los pacientes con DM2 sobre cambios de atención terciaria a primaria

Diseño: forma parte de un estudio de intervención. Cualitativo.

Métodos: entrevistas narrativas a 44 pacientes suecos.

Diseño: cualitativo. los encuentros médicos en
Diseño: fenomenología inductiva.

Métodos: observación y entrevistas a 18 participantes suecos de atención primaria. manejo de la enfermedad.

La falta de motivación para llevar a cabo un adecuado automanejo así como una falta de tangibilidad en las recomendaciones sanitarias han sido vistas por los pacientes con DM2 como las principales barreras para manejar la enfermedad.

Necesidad de información de calidad.

Falta de conocimiento del porqué de las recomendaciones y malentendidos sobre las recomendaciones dietéticas afectan al automanejo.

Diferencias entre profesionales y pacientes.

No compartir mismos objetivos, actitud autoritaria, falta de competencia profesional dificultan el automanejo.

Métodos: entrevistas niveles asistenciales.

semi-estructuradas a 12 Falta de continuidad entre niveles participantes de un cen- asistenciales. Importancia en la tro de salud de atención longitud e intimidad de consultas. primaria. Prefieren la atención recibida en el centro de salud.

\begin{tabular}{|c|c|c|c|c|}
\hline $\begin{array}{l}\text { Jones RA; } \\
\text { Utz SW, } \\
\text { Williams IC, } \\
\text { Hinton I, } \\
\text { Alexander G, } \\
\text { Moore C } \\
\text { y col.; 2008 }\end{array}$ & $\begin{array}{l}\text { Estados } \\
\text { Unidos }\end{array}$ & $\begin{array}{l}\text { Examinar el impacto de la } \\
\text { familia en el manejo de la } \\
\text { DM2 }\end{array}$ & $\begin{array}{l}\text { Diseño: cualitativo. } \\
\text { Métodos: entrevistas na- } \\
\text { rrativas a } 21 \text { pacientes } \\
\text { afro-americanos de aten- } \\
\text { ción primaria. }\end{array}$ & $\begin{array}{l}\text { Ambivalencia en el soporte brin- } \\
\text { dado por la familia. } \\
\text { Familia: Soporte positivo y nega- } \\
\text { tivo al mismo tiempo. Aunque en } \\
\text { ocasiones la familia sea útil, en } \\
\text { otras, crean un ambiente que di- } \\
\text { ficulta el automanejo de la enfer- } \\
\text { medad. }\end{array}$ \\
\hline $\begin{array}{l}\text { Lai W.A, } \\
\text { Lew-Ting C.Y, } \\
\text { Chie W.C; } \\
2005^{20}\end{array}$ & Taiwan & $\begin{array}{l}\text { Describir las percepciones } \\
\text { sobre la enfermedad de pa- } \\
\text { cientes de China con DM2 } \\
\text { y las estrategias de auto- } \\
\text { cuidado }\end{array}$ & $\begin{array}{l}\text { Diseño: cualitativo. } \\
\text { Métodos: entrevistas en } \\
\text { profundidad a } 22 \text { parti- } \\
\text { cipantes (12 hombres y } \\
10 \text { mujeres) de atención } \\
\text { primaria. }\end{array}$ & $\begin{array}{l}\text { La falta de conocimientos afecta } \\
\text { al automanejo. } \\
\text { Los participantes perciben la dieta } \\
\text { como fundamental para el automa- } \\
\text { nejo. Aunque sus conocimientos } \\
\text { en relación a la dieta son incom- } \\
\text { pletos. }\end{array}$ \\
\hline $\begin{array}{l}\text { Lawton J, } \\
\text { Peel E, } \\
\text { Parry 0, } \\
\text { Araoz G, } \\
\text { Douglas M; } \\
\text { 2005 }^{52}\end{array}$ & Escocia & $\begin{array}{l}\text { Valorar las percepciones } \\
\text { de diabéticos recién diag- } \\
\text { nosticados sobre la enfer- } \\
\text { medad y sobre los servi- } \\
\text { cios de salud }\end{array}$ & $\begin{array}{l}\text { Diseño: cualitativo explo- } \\
\text { ratorio. } \\
\text { Métodos: entrevistas en } \\
\text { profundidad a } 40 \text { pacien- } \\
\text { tes hospitalizados y de } \\
\text { atención primaria. }\end{array}$ & $\begin{array}{l}\text { Malentendidos en referencia a los } \\
\text { niveles asistenciales. } \\
\text { Conocimientos erróneos sobre los } \\
\text { niveles asistenciales que les influ- } \\
\text { ye en el concepto de su enferme- } \\
\text { dad y en el automanejo. }\end{array}$ \\
\hline
\end{tabular}




\begin{tabular}{|c|c|c|c|c|}
\hline Artículo & País & Objetivo & Metodología & Resultados más relevantes \\
\hline $\begin{array}{l}\text { Lawton J, } \\
\text { Rankin D, } \\
\text { Peel E, } \\
\text { Douglas M; } \\
\text { 2009 }^{52}\end{array}$ & Escocia & $\begin{array}{l}\text { Examinar las experiencias } \\
\text { de los pacientes con DM2 } \\
\text { sobre la reestructuración } \\
\text { de los servicios sanitarios }\end{array}$ & $\begin{array}{l}\text { Diseño: cualitativo. } \\
\text { Métodos: entrevistas en } \\
\text { profundidad a } 20 \text { pacien- } \\
\text { tes hospitalizados y de } \\
\text { atención primaria. }\end{array}$ & $\begin{array}{l}\text { Malentendidos en referencia a } \\
\text { los niveles asistenciales. } \\
\text { Malentendidos por los motivos de } \\
\text { traspaso de la atención entre ni- } \\
\text { veles asistenciales. Percepción de } \\
\text { que sí son atendidos en el centro } \\
\text { de salud su enfermedad está bajo } \\
\text { control y sin riesgo de complica- } \\
\text { ciones graves. }\end{array}$ \\
\hline
\end{tabular}

\begin{tabular}{llll}
\hline Lippa KD, & Estados & Examinar las experiencias & Diseño: cualitativo. \\
Klein HA; & Unidos & de pacientes con DM2 so- & Métodos: entrevistas semi- \\
2008 $^{19}$ & & bre aspectos relacionados & estructuradas a 18 par- \\
& & con su automanejo & ticipantes de atención \\
& & primaria.
\end{tabular}

La incomprensión afecta al automanejo de la DM2.

Falta de conocimiento en las principales áreas que influyen en un automanejo adecuado (enfermedad, dieta, ejercicio, etc).

$\begin{array}{llll}\text { Lohri-Posey B; } & \text { Estados } & \text { Describir las experiencias } & \text { Diseño: fenomenológico. } \\ \mathbf{2 0 0 6}^{\mathbf{3 5}} & \text { Unidos } & \text { de pacientes con DM2 que } & \text { Métodos: entrevistas en } \\ & & \text { viven en un entorno rural } & \text { profundidad a 13 pacien- } \\ & \text { de los Apalaches y con ba- } & \text { tes de } 2 \text { clínicas del no- } \\ & \text { jos ingresos } & \text { roeste de Virginia. }\end{array}$

Ambivalencia en el soporte brindado por la familia.

Familia como soporte (en la toma de medicación y dieta adecuada) y como estresor (las mujeres producen miedo a sus cónyuges en relación a los peligros físicos que conlleva convivir con la DM2). Necesidad de formación en prevención en sus descendientes. Lundberg PC, Tailandia
Thrakul S;
2012 $^{34}$
Describir como los pa- Diseño: cualitativo descientes budistas con DM2 criptivo. practican y describen el automanejo
La tradición cultural, la religión Métodos: observación y la economía afecta al automaneentrevistas semi-estruc- jo. La familia principal soporte turadas a 30 pacientes social.

con DM2 originarios de Tailandia.

Diseño: cualitativo, ex- Barreras y estrategias para auploratorio, descriptivo. tomanejo. Barreras: Falta de coMétodos: grupos focales nocimiento sobre el plan dietétia 24 pacientes de centro co. Estrategias: Desarrollar una de salud rural. relación de colaboración con el profesional.

\begin{tabular}{|c|c|c|c|c|}
\hline $\begin{array}{l}\text { Oftedal B, } \\
\text { Karlsen B, } \\
\text { Bru E; } 2010^{49}\end{array}$ & Noruega & $\begin{array}{l}\text { Describir las percepciones } \\
\text { de pacientes con DM2 so- } \\
\text { bre soporte recibido por } \\
\text { profesionales sanitarios } \\
\text { y como este influye en el } \\
\text { automanejo }\end{array}$ & $\begin{array}{l}\text { Diseño: cualitativo inter- } \\
\text { pretativo y descriptivo. } \\
\text { Métodos: } 3 \text { grupos fo- } \\
\text { cales. } 19 \text { pacientes con } \\
\text { DM2. }\end{array}$ & $\begin{array}{l}\text { Necesidades no cubiertas. } \\
\text { Necesidad de consejo práctico en } \\
\text { la consulta sobre dieta, ejercicio } \\
\text { y de soporte de grupo educativo } \\
\text { continuado. }\end{array}$ \\
\hline $\begin{array}{l}\text { Pilkington FB, } \\
\text { Daisuki I, } \\
\text { Bryant T, } \\
\text { Dinca- } \\
\text { Panaitescu M, } \\
\text { Dinca- } \\
\text { Panaitescu S, } \\
\text { Raphael D; } \\
\mathbf{2 0 1 0}^{43}\end{array}$ & Canadá & $\begin{array}{l}\text { Comprender como pacien- } \\
\text { tes con DM2 con bajos in- } \\
\text { gresos económicos se ven } \\
\text { afectados, desde su expe- } \\
\text { riencia en el automanejo }\end{array}$ & $\begin{array}{l}\text { Diseño: cualitativo. } \\
\text { Métodos: entrevistas semi- } \\
\text { estructuradas a } 60 \text { pa- } \\
\text { cientes con DM2 de } 4 \\
\text { centros de atención pri- } \\
\text { maria en Toronto. }\end{array}$ & $\begin{array}{l}\text { La importancia de la economía } \\
\text { en el automanejo. } \\
\text { La pobreza influye en el control } \\
\text { glicémico, en la dieta y en el au- } \\
\text { tomanejo y es un factor de riesgo } \\
\text { para padecer la DM2. } \\
\text { La economía de los pacientes no } \\
\text { les permite comprar los alimen- } \\
\text { tos más adecuados, ni todos los } \\
\text { medicamentos que su médico les } \\
\text { prescribe. }\end{array}$ \\
\hline
\end{tabular}




\begin{tabular}{lllll}
\multicolumn{1}{c}{ Artículo } & \multicolumn{1}{c}{ País } & \multicolumn{1}{c}{ Objetivo } & \multicolumn{1}{c}{ Metodología } & \multicolumn{1}{c}{ Resultados más relevantes } \\
\hline Ramsay & Estados & Explorar como los pacien- & Diseño-enfoque: cualitati- & Barreras para automanejo. \\
Wan C, Vo L, & Unidos & tes perciben el empodera- & vo Inductivo. & Rol pasivo en automanejo. Barre- \\
Barnes CS; & & miento, las interaciones & Métodos: entrevistas & ras internas (impulsos, frustra- \\
$\mathbf{2 0 1 2}^{46}$ & & con los profesionales y & semi-estructuradas a 29 & ción relacionada con el padecer \\
& & comportamientos sobre el & pacientes con DM2 de la enfermedad y fatiga) y externas \\
& & automanejo & una clínica del sector & (nivel económico y limitación del \\
& & público. & tiempo en los encuentros médicos) \\
& & & para un adecuado automanejo. \\
\hline
\end{tabular}

\begin{tabular}{lll}
\hline Song Y, & Estados & Identificar las fuentes pri- \\
Song HJ, & Unidos & marias de soporte social \\
Han HR, & & de koreanos que viven en \\
Park SY, & & América yer cómo afec- \\
Nam S, & tan al automanejo de la \\
Kim MT; & DM2 \\
$\mathbf{2 0 1 2}^{\mathbf{3 1}}$ &
\end{tabular}

Diseño: cuantitativo.
Guiados por la teoría so-
cial cognitiva.

Métodos: cuestionarios a

83 participantes con DM2

Koreanos que viven en América. SDSCA(valora actividades de automanejo), SCDSE (valora la auto-eficacia), soporte social se valoró con escala validada de tipo Likert.

\begin{tabular}{lll}
\hline Sowattanangoon & Tailandia & Explorar cómo los pacien- \\
N, Kotchabhakdi & & tes tailandeses con DM2 \\
N, Petrie K.J; & & perciben y manejan su en- \\
$\mathbf{2 0 0 9}^{38}$ & fermedad
\end{tabular}

Diseño: etnográfico.

Métodos: entrevistas semi-estructuradas a 27 pacientes con DM2 de dos hospitales públicos en Bangkok.

Soporte necesario para un correcto automanejo.

Principal soporte social es el cónyuge. Variabilidad entre sexos.

Mujeres más necesidad de soporte que los hombres.

Necesidad de soporte emocional, actividad física, dieta y cuidado de píes.

Importancia de la multicultura.

Percepción de la enfermedad errónea. La dieta está influenciada por la cultura budista y afecta a un adecuado automanejo de la enfermedad. La religión les supone un apoyo para automanejar su enfermedad.

\begin{tabular}{|c|c|c|c|}
\hline $\begin{array}{l}\text { Sturt J, } \\
\text { Hearnshaw H, } \\
\text { Barlow J.H, } \\
\text { Hainsworth J, } \\
\text { Whitlock S; } \\
\text { 2005 }^{21}\end{array}$ & Reino Unido & $\begin{array}{l}\text { Identificar las necesidades } \\
\text { educativas y de soporte } \\
\text { para el automanejo desde } \\
\text { la perspectiva de pacien- } \\
\text { tes }\end{array}$ & $\begin{array}{l}\text { Diseño: cualitativo. } \\
\text { Métodos: grupos focales } \\
\text { a } 23 \text { participantes de } \\
\text { atención primaria. }\end{array}$ \\
\hline
\end{tabular}

Necesidades de información no cubiertas.

Necesidad de conocimientos sobre el porqué de las recomendaciones. Necesidad de conocer la interpretación de los niveles de azúcar en sangre para mantener un adecuado automanejo.

\begin{tabular}{llll}
\hline Wang Y, & Estados & Identificar los conocimien- & Diseño: cualitativo. \\
Chuang L, & Unidos & tos, herramientas y expe- & Métodos: grupos focales \\
Bateman WB; & & riencias relacionadas con a 15 participantes con \\
$\mathbf{2 0 1 2}^{39}$ & & el automanejo de la pobla- & DM2 Chinos que viven en \\
& & ción china con DM2 & América.
\end{tabular}

Importancia de la multicultura.

La cultura influye en un adecuado automanejo. Falta de conocimientos relacionados con la dieta. La falta de material educativo en su idioma de origen es percibido como barrera para automanejo.

\begin{tabular}{ll}
\hline Weiler DM, & Estados \\
Crist JD; & Unidos \\
2009 $^{\mathbf{4 0}}$ &
\end{tabular}

Explorar las influencias socio-culturales y el contexto social de inmigrantes latinos
Diseño: descriptivo. Teoría fundamentada

Métodos: entrevistas semiestructuradas a 10 participantes con DM2 (6 mujeres y 4 hombres) latinos que viven en EEUU.
Importancia de la multicultura.

El estigma, la cultura y la percepción social de la enfermedad como una patología maligna y ocultada en el entorno influyen en el automanejo de la enfermedad. Cohesión familiar pilar importante. 
Después de la crítica y lectura de los artículos, 5 temas emergieron y se presentan a continuación:

1. Atención sanitaria transcultural: prioritaria para un adecuado automanejo.

2. La familia: soporte importante para el automanejo de la DM2.

3. Barreras y facilitadores para el automanejo de la DM2.

4. La crisis económica, obstáculo importante para un correcto automanejo.

5. El sistema sanitario y los profesionales: ¿estamos respondiendo las necesidades de los pacientes para que manejen la DM2?

\section{Atención sanitaria transcultural: prioritaria para un adecuado automanejo}

La cultura es un pilar importante en la vida de las personas e influye en todas las esferas del ser humano ${ }^{33}$. En esto se apoya la teoría transcultural de Madeleine Leininger. Según esta autora, es imprescindible que los profesionales de enfermería se formen en el análisis de las diferentes culturas y subculturas del mundo, cuyo propósito consiste en concebir un saber científico y humanístico para que proporcione una práctica de cuidados enfermeros específicos para cada cultura ${ }^{33}$.

En esta revisión, han sido 8, los estu$\operatorname{dios}^{23,34-40}$ que han abordado el tema de la cultura y cómo esta influye en el automanejo de la DM2. La homeopatía y las hierbas medicinales continúan emergentes en el tratamiento coadyuvante de la enfermedad. Así se recoge en los estudios realizados por Fleming y col $^{37}$, donde estudiaron la cultura musulmana, en el de Wang y col $^{39}$, donde analizaron la cultura china y en el realizado por Lundberg y $\mathrm{col}^{34}$ con pacientes budistas. En estos estudios identificaron cómo para los pacientes, las medicinas alternativas suponían un apoyo importante para prevenir complicaciones severas relacionadas con los efectos secundarios de la medicación actual. Otro aspecto impor- tante que se ha identificado en la revisión, es cómo los pacientes latinos perciben el lenguaje como una barrera lingüística que influye en el automanejo de la DM2. De este modo se refleja en el estudio llevado a cabo por Caban y $\mathrm{col}^{36}$, donde pacientes latinos se sentían discriminados en cuanto a barreras de lenguaje con los profesionales. La falta de material en la lengua china dificultaba el entendimiento y en definitiva el automanejo del paciente chino inmigrante $^{39}$. Además, el contenido del material educativo no estaba adaptado a las diferentes culturas $^{23}$.

Por otro lado, la dieta es uno de los pilares fundamentales en la cultura. Por ello, 6 estudios $^{23,36-40}$ han abordado este tema. El rol social del hombre musulmán respecto a comer más cantidad que la mujer en festejos sociales llevaba a una alteración del orden normal en su proceso de automanejo de la DM2 $2^{27}$. Weiler y col $^{40}$ destacaron la ausencia de los pacientes latinos a celebraciones sociales debido a que las comidas no eran adecuadas para su enfermedad. El arroz, principal alimento en la población latina y china, suponía una barrera que debían aprender a superar por su alto contenido en carbohidratos ${ }^{23,34,36,38}$.

A todo lo anterior, se suma otro tema muy presente en cada cultura, la religión. Cuatro fueron los estudios ${ }^{23,34,36,38}$ que exploraron la relevancia de la religión en el automanejo de la patología. Tanto la causa como el manejo de la enfermedad estaba vinculada a su religión y a la fe en su $\operatorname{Dios}^{23,34,36,38}$. Así fue recogido en el estudio llevado a cabo por Sowattanangoon y col $^{38}$ con pacientes procedentes de Thailandia que practicaban el budismo como religión donde identificaron en sus pacientes la percepción de que su Dios le había "regalado" esta enfermedad, y en el trabajo desarrollado por Adams ${ }^{23}$ donde las participantes latinas se ayudaban de su fe para disponer de la fuerza necesaria para manejar su enfermedad.

Asimismo, el rol social de multicuidador que tenía la mujer africana dificultaba el ser ayudada por su familia en relación a su automanejo ${ }^{41}$. 


\section{La familia: soporte importante para el automanejo de la DM2}

La familia es reconocida por los pacientes como un soporte social importante como apoyo físico o instrumental, mental y financiero. Así se recoge en los trabajos realizados por Abbott y $\mathrm{col}^{22}$, Lundberg y col$^{34}$, Lohry-Posey ${ }^{35}$, Burke y col ${ }^{42}$ y Pilkington $\mathrm{y} \mathrm{col}^{43}$. Estos estudios describieron una ayuda referida al plan dietético. En concreto, Burke y col ${ }^{42}$ identificaron en los pacientes varones un apoyo por parte de sus esposas para comer aquello que era adecuado a su enfermedad, motivándoles a evitar las tentaciones. Asimismo, las mujeres recibían ayuda de sus maridos con recordatorios de la medicación y favoreciendo la comunicación con los profesionales. Además, Lundberg y $\mathrm{col}^{34}$ destacaron el rol de la familia a la hora de acompañar a los pacientes a las citas médicas.

Por el contrario, se identificaron cuatro estudios ${ }^{23,35,41,44}$, donde se mostraron resultados contradictorios. Según Jones y $\mathrm{Col}^{44}$ los pacientes percibían este soporte como positivo y negativo al mismo tiempo ya que la familia tenía pocos conocimientos sobre la enfermedad y sobre las necesidades específicas de los pacientes ${ }^{44}$. Lohry-Posey ${ }^{35}$ describió que las esposas llegaban a causar estrés en los maridos con DM2, al inculcarles miedo para evitar que realizaran trabajos costosos que supusieran un peligro para su integridad física como por ejemplo un corte en el puesto de trabajo. Por último, Adams ${ }^{23}$ identificó una incomprensión por parte de la familia debida al desconocimiento de lo que significaba para el paciente convivir con la enfermedad.

Cuando la familia se convertía en un soporte negativo para el paciente, producía una sumisión y una disminución de su independencia a la hora de favorecer y continuar con su correcto automanejo ya que sustituían a los pacientes en el automanejo ${ }^{41}$.

\section{Barreras y facilitadores para el automanejo de la DM2}

Handley y col $^{45}$ identificaron la falta de motivación, tangibilidad y evidencia de las recomendaciones sanitarias como las principales barreras. Al mismo tiempo, Ramsay $\mathrm{y} \mathrm{col}^{46}$ en su estudio, dividieron las barreras en internas y externas. En referencia a las barreras internas, se identificaron la fatiga producida por los medicamentos y la frustración de padecer la enfermedad. Los problemas económicos, las barreras lingüísticas, los efectos secundarios de la medicación y la limitación en el tiempo referido a los encuentros médicos para realizar preguntas, fueron vistos como las principales barreras externas ${ }^{46}$. Las barreras lingüísticas y la falta de contenido educativo adaptado a la cultura propia de los pacientes, fueron considerados impedimentos importantes según el estudio desarrollado por Adams ${ }^{23}$

Por otro lado, el ejercicio se veía dificultado por las consecuencias propias de padecer la DM2 como por ejemplo, la existencia de problemas cardiacos a consecuencia de la progresión de la enfermedad, como la fatiga o una visión más afectada ${ }^{42}$.

Nagelkerk y $\operatorname{col}^{47}$ llevaron a cabo un estudio descriptivo exploratorio para analizar las barreras y elementos facilitadores que los pacientes identificaron para desarrollar un adecuado automanejo de la DM2. En referencia a las barreras, se describieron la falta de entendimiento sobre un plan dietético, la falta de un cuidado individualizado, recursos económicos limitados, la inespecificidad de las sesiones educativas de grupo al estar muy estandarizadas y la falta de coordinación entre los diferentes ámbitos asistenciales ${ }^{47}$. Por otro lado, el mantener una actitud positiva ante la enfermedad, la disponibilidad de recursos necesarios para poder automanejar su patología, el mantenimiento de una rutina en el horario en relación a la toma de medicacióny la asistencia a grupos educativos efectivos que incluyeran una demostración práctica dirigido por educadores cualificados, fueron considerados los principales facilitadores para llevar a cabo un adecuado automanejo ${ }^{47}$. 


\section{La crisis económica, obstáculo importante para un correcto automanejo}

La crisis económica mundial que estamos atravesando desde hace varios años afecta al paciente diabético y a su automanejo. Así se recoge en 5 estudios ${ }^{23,34,43,47,48}$ encontrados en la literatura que abordaban la economía como aspecto clave para un adecuado automanejo. Según el trabajo desarrollado por Lundberg y col ${ }^{34}$ la prioridad de los pacientes era llevar dinero a casa por medio del trabajo, lo que implicaba menos tiempo para preparar comidas adecuadas a su enfermedad decantándose por comidas preparadas como chocolatinas o pizzas. Varios autores advirtieron de la dificultad que expresaban los pacientes para adquirir la comida más adecuada, por ser poco económica y por anteponer a sus hijos antes que a su enfermedad, comprando alimentos básicos para ellos ${ }^{23,43,47}$. Además, Nagelkerk y $\mathrm{col}^{47}$ y Pilkington y $\mathrm{col}^{43}$ subrayaron que el coste elevado de la medicación y el tener más medicamentos prescritos llevaba a los pacientes a no tomarse su medicación a diario. Asimismo, la dificultad económica de los pacientes hacía difícil el control glicémico por falta de glucómetro en sus hogares $^{23,43,47}$. Por último, es importante destacar que Pilkington y $\mathrm{col}^{43}$ identificaron en sus participantes la dificultad de comprarse unos calzados apropiados para sus pies porque les urgía más comprar ropa para sus hijos. El acceso a especialistas como el podólogo era poco común en aquellas clases con recursos económicos menores ya que dependía de la situación económica de cada paciente para hacerse con un seguro sanitario adecuado ${ }^{23,48}$.

\section{El sistema sanitario y los profesionales: ¿estamos respondiendo a las necesidades de los pacientes para que manejen la DM2?}

Qué tipo de información y recursos demandan los pacientes con DM2 a sus profesionales sanitarios?

Los pacientes con DM2 demandaban a sus profesionales recursos que ayudasen a aplicar la información recibida en su día a día con respecto a la dieta, el ejercicio y el control glicémico ${ }^{21}$. Sturt y col $^{21}$, en este estudio, identificaron en los pacientes una falta de entendimiento de las recomendaciones dadas, refiriendo cierta inconsistencia en la información proveniente de los diversos profesionales sanitarios que les atendieron. Además, destacaron la importancia del apoyo grupal para impulsar el ejercicio y manifestar sus dudas con pacientes con la misma patología. Esta necesidad también fue identificada en el estudio desarrollado por Oftedal y col ${ }^{49}$ donde utilizaron los grupos focales como método de recogida de datos. Oftedal y col $^{49}$ describieron que era necesario una complementación entre la competencia profesional del personal sanitario y el conocimiento del propio paciente, ya que era el propio enfermo el experto en relación a su enfermedad. El profesional debía motivar y cuando esta asociación se rompía, el paciente podía reducir sus esfuerzos para continuar manejando su DM2 y acabar rechazando al profesional ${ }^{43}$.

Abbott y col ${ }^{22}$, en su estudio, reafirmaron la necesidad que tenían los pacientes de conocer los motivos de las recomendaciones dadas por los profesionales así como la necesidad de un vocabulario menos técnico. Asimismo, se demandaron más visitas domiciliarias y más y mejor acceso al especialista.

Por otro lado, los participantes de la investigación llevada a cabo por Burke y $\mathrm{col}^{42}$ expresaron la necesidad de que los profesionales escucharan sus experiencias y dificultades para llevar a cabo su automanejo, dedicando más tiempo del que hasta ahora se les brindaba y que al mismo tiempo reconocieran los pequeños logros que estaban consiguiendo. Además, información sobre otras fuentes como el dietista, fueron descritas como prioritarias para los pacientes ${ }^{42}$.

A pesar de la demanda de información, los pacientes requerían que esa información se proporcionara de manera respetuosa y progresiva, evitando los mensajes alarmistas ${ }^{23}$.

Asimismo, Song y $\mathrm{col}^{31}$, en su estudio, obtuvieron diferencias entre sexos en re- 
lación a las necesidades de información y de soporte. Concluyeron que sus participantes requerían información y soporte en relación al control emocional, a qué tipo de actividad física debían de realizar, a cuáles eran las recomendaciones dietéticas a llevar a cabo y a cuáles debían ser los cuidados en el control de pies, en el control glicémico y en la toma de medicación. Aún así, fueron las mujeres quienes más necesidad de información y de soporte expresaron $^{31}$.

En consonancia, Sturt y col $^{21}$ añadieron que los pacientes con DM2 veían la necesidad de comprender los parámetros óptimos de la glucosa en sangre y la medida e impacto de la descompensación de la glucosa. Igualmente vieron ineludible comprender el motivo por el que requerían medicación, el conocimiento de su mecanismo de acción y cómo actuaba la dieta y el ejercicio en la reducción de los niveles de azúcar en sangre. Lippa y col $^{19}$ corroboraron estas necesidades y añadieron que los pacientes sabían que debían tomar la medicación pero desconocían su función, cómo manejar las dosis omitidas, el objetivo de la monitorización de la glucemia y la relación entre el ejercicio y el control glicémico. Además, no eran capaces de detectar los síntomas de híper e hipo glucemias ${ }^{19}$.

La confusión en cuanto a qué alimentos estaban permitidos y cuáles no fue un tema presente en la investigación realizada por Wang y col ${ }^{39}$ puesto que los pacientes de su estudio no comprendían por qué el azúcar proveniente de la fruta era aconsejable y el de otros alimentos no. De la misma forma, existía aún una necesidad de información sobre las causas y las complicaciones de la enfermedad ${ }^{18,43}$.

Otro problema real es que los pacientes conocen las recomendaciones pero carecen de información con respecto a los motivos de esos consejos sanitarios;esto produce en ellos una disminución de la responsabilidad de manejar su enfermedad. Así fue corroborado por Holmström y col ${ }^{18}$ y Abbott y col $^{22}$.

Finalmente, en varios estudios, destacaron la importancia de la continuidad de cuidados en el fomento del automanejo de la DM2. Concretamente, se resaltó la importancia de ser recibidos por el mismo profesional y la necesidad de eliminar las barreras estructurales existentes entre niveles asistenciales para así aumentar la comunicación entre profesionales $\mathbf{s}^{22,42,48,49}$.

¿Cómo deben desarrollarse las consultas sanitarias para promover un correcto automanejo de la DM2?

Hornsten y $\mathrm{col}^{50}$ fueron muy explícitos en el estudio que llevaron a cabo, al identificar qué actitudes del profesional sanitario en las consultas eran inadecuadas para fomentar un adecuado automanejo de la enfermedad. Concretamente, destacaron como estrategias poco efectivas centrar las consultas en resultados o datos exclusivamente biomédicos como el control glicémico o la fisiopatología de la enfermedad o intentar fomentar por medio del autoritarismo la aceptación y puesta en marcha de las recomendaciones dadas por el profesional $^{50}$. Esto fue corroborado por Oftedal y $\mathrm{col}^{49}$, donde identificaron que los pacientes de su estudio necesitaban, además, que el personal sanitario tratara y se preocupara por la totalidad de la persona a la que atendía mediante un trato empático.

¿Somos los profesionales sanitarios conscientes de que existe unos conocimientos erróneos en nuestros pacientes y de que esto influye en el correcto desarrollo y funcionamiento del automanejo de la DM2?

La percepción de que los pacientes con DM2 disponen de conocimientos erróneos es una realidad. Así fue comprobado por Lai y $\mathrm{col}^{20}$, al identificar en sus participantes unos conocimientos erróneos o incompletos en relación a las recomendaciones dietéticas. Los pacientes del estudio desarrollado por Lai y $\mathrm{col}^{20}$ aseguraron que únicamente los dulces eran perjudiciales para su salud en relación a su enfermedad. Otros pacientes, conforme al trabajo desarrollado por Holmström y col ${ }^{18}$, expresaron que el alimento prohibido para un adecuado automanejo de la DM2 era la grasa en su conjunto. Del mismo modo, Lippa y $\mathrm{col}^{19}$, aseguraron que algunos de sus participantes consideraron que las labores domésti- 
cas era suficiente ejercicio para manejar su patología. Lundberg y $\mathrm{col}^{34}$ se sumaron y añadieron que los participantes de su estudio si no tenían síntomas de la DM2 no controlaban ni manejaban su enfermedad al no tomar las medicinas recetadas por su médico y continuaban realizando las actividades que hasta el momento de padecer la patología realizaban, como ingerir alcohol. Del mismo modo, Sowattanangoon y $\mathrm{col}^{38}$ expusieron que sus participantes y familias percibían que la DM2 estaba relacionada con el azúcar en la orina. Y esta percepción errónea, según concluyeron estos autores, cambiaba la mira de los objetivos de los pacientes en referencia a su automanejo.

Asimismo, que los pacientes calificaran la DM2 como reversible y de poca relevancia al afirmar que la relación con su profesional iría disminuyendo conforme fueran desapareciendo los síntomas clínicos, fue descrito por Lawton y col $^{51}$. Adams ${ }^{23}$, llegó más lejos al afirmar en su estudio que algunos de sus participantes percibían la enfermedad como trasmisible. Los participantes del estudio llevado a cabo por Holmström $\mathrm{y} \mathrm{col}^{18}$ describieron que la DM2 no era una enfermedad real porque carecían de síntomas ocomplicaciones.

Las creencias religiosas, en ocasiones, producen en el paciente una mentalidad errónea en relación a la percepción de reversibilidad de la enfermedad. Handley y $\mathrm{col}^{45}$ identificaron que los pacientes de su estudio estaban convencidos de que su Dios ayudaría a revertir las complicaciones que fueran adquiriendo.

Finalmente, se debe citar la falta de conocimientos en referencia a los motivos de traspaso de atención terciaria a primaria ya que los pacientes del estudio llevado a cabo por Lawton y col ${ }^{51}$ y Lawton y col${ }^{52}$ lo relacionaban con una mejoría de su enfermedad.

\section{DISCUSIÓN}

A través de esta revisión, se ha podido conocer aspectos clave para implantar un adecuado automanejo de la DM2 en los servicios socio sanitarios españoles. Estas indicaciones pueden ser de gran relevan- cia teniendo en cuenta la dirección que las Estrategias de la Cronicidad ${ }^{53}$ y de la Diabetes $^{54}$ están tomando en los últimos años hacia el fomento de la autonomía y autocuidado del paciente. Del mismo modo, los temas identificados en esta revisión se consideran de gran utilidad para poder desarrollar un programa futuro que garantice un adecuado automanejo a la población española afectada por esta patología.

Con respecto a los estudios incluidos en este trabajo, se debe resaltar que disponen de diseños muy completos aunque principalmente de índole cualitativa (Teoría fundamentada, Fenomenología hermenéutica y diseño etnográfico). Se debe, de la misma manera, destacar la falta de artículos que incluyan intervenciones porque esta revisión bibliográfica se ha centrado en la exploración de las necesidades de los pacientes con DM2 y no en las intervenciones o su eficacia sobre las necesidades de los pacientes. Esto es, el objetivo de este trabajo es conocer qué necesitan los pacientes con DM2 para poder abordar en un futuro dichos programas. No se han encontrado estudios llevados a cabo en el ámbito español y por ello, para aplicar los resultados de esta revisión, se recomienda tener en cuenta las diferencias contextuales, de políticas de salud y culturales de los diferentes países.

Se debe resaltar la gran relevancia que tienen los resultados de la revisión en relación a la atención sanitaria transcultural, teniendo en cuenta la situación actual de España con el incremento de la población inmigrante y la diversidad cultural. Para proporcionar una atención integral a las personas es necesario conocer su cultura. Además, la falta de material educativo en los diferentes idiomas y la falta de atención al ámbito cultural del paciente, han sido vistas como barreras importantes para mantener un adecuado automanejo de la DM2 $2^{39,42}$. Por ello y puesto que este es un tema emergente, se debe considerar para posteriores estudios de investigación y para el desarrollo de futuros programas de automanejo que se implementen en España.

Del mismo modo, la familia, ha sido uno de los temas presentes en la revisión. En la 
cultura española ésta sigue siendo uno de los principales soportes emocionales, instrumentales y financieros de los pacientes para la consecución de un correcto automanejo de la enfermedad ${ }^{22,37,38,45,46}$. A pesar de ello, es patente la falta de protagonismo que el equipo investigador y el sistema sanitario brinda a la familia ya que no se han encontrado estudios en esta revisión que les incluyan como participantes. Y esto, tiene que ser valorado para futuros programas $o$ intervenciones que se vayan a implementar.

La crisis económica, principal problema que está atravesando en la actualidad España y otros países europeos, afecta, como bien se ha visto en este trabajo, al correcto automanejo de la DM2. A pesar de ello, se debe considerar que la información hallada no puede ser extrapolable al contexto español porque la mayoría de pacientes disponen de sanidad pública a diferencia de los participantes de varios de los trabajos revisados. Pero estos resultados pueden ser más relevantes en el caso de los pacientes inmigrantes que no poseen la tarjeta individual sanitaria y que residen en nuestro país.

Las barreras para el desarrollo del automanejo de la DM2 halladas en la revisión son motivadas en su mayoría por la falta de entendimiento sobre aspectos relacionados con la enfermedad, por la falta de atención a la cultura, al idioma y por actitudes equivocadas del profesional sanitario en las consultas con su paciente. Posibles soluciones y explicaciones a estas barreras se han abordado en la sección de respuesta a las necesidades de este mutuo trabajo. Se ha manifestado que las necesidades de información, culturales y económicas de las personas que padecen DM2 no quedan reflejadas en la atención sanitaria. Esto lleva a reflexionar si realmente las intervenciones o programas que se están implementando están centradas en el automanejo del paciente, considerando sus expectativas, posibilidades y prioridades o por el contrario, se centran únicamente en el manejo biomédico de la enfermedad ${ }^{49}$. Asimismo, es importante que los profesionales tomen conciencia de que los mensajes alarmistas y persuasivos no llevan sino a un empeoramiento y deterioro del proceso relacional paciente-profesional con un detrimento significativo en el desarrollo de un automanejo adecuado. Igualmente, los pacientes demandan al personal sanitario un mayor empoderamiento e implicación en su proceso de salud-enfermedad, enseñándoles no sólo aquello que deben realizar sino también el motivo de dicha recomendación ${ }^{18,22}$. Además, queda patente en este trabajo que los pacientes no muestran tanto interés en conocer los aspectos más biomédicos de la enfermedad sino, en la mejor manera de integrar la DM2 en su día a día, mantener la normalidad y manejar los cambios producidos ${ }^{21,52}$.

En esta línea, este trabajo es importante para que los profesionales sanitarios tomen conciencia de lo que el paciente, protagonista de su enfermedad, necesita y requiere. Finalmente, para el desarrollo e implementación de un futuro programa de automanejo o autocuidado se requiere, en primer lugar, concienciar a los profesionales sanitarios de las necesidades y preferencias de los pacientes. Esto resulta esencial para poder fomentar la asociación paciente-profesional que los programas de automanejo/autocuidado requieren. Es importante, del mismo modo, erradicar las actitudes paternalistas de los profesionales sanitarios hacia los pacientes ya que el respeto, la comprensión y motivación pueden resultar claves en estos casos. De igual manera, estos programas deben ser realistas y en la actualidad es esencial que reflejen la situación actual de la sociedad española en cuanto a la multiculturalidad y la dificultad económica. Por último, y no menos importante, cualquier intervención que se vaya a desarrollar con el paciente con DM2 debe tener en consideración a la familia. Como se ha mostrado en este trabajo, el paciente diabético considera a la familia un soporte social, educativo, cultural, emocional y económico importante en el manejo de la DM2 $22,37,38,45,46$.

\section{Agradecimientos}

Al Equipo del proyecto MEDEA del País Vasco, por calcular el índice de privación $\mathrm{y}$ aportar datos del índice, y en especial a Montse Calvo, por el trabajo de geocodificación de los datos. 


\section{BIBLIOGRAFÍA}

1. Baumann LC, Opio CK, Otim M, Olson L, Eluison S. Self-care beliefs and behaviors in Ugandan adults with type 2 diabetes. Diabet Educ 2010; 36: 293-300.

2. Powell CK, Hill EG, Clancy DE. The relationship between health literacy and diabetes knowledge and readiness to take health actions. Diabet Educ 2007; 33: 144-151.

3. Organización Mundial de la Salud (OMS) (sitio en Internet). Centro de prensa. Diabetes. Disponible en: http://www.who.int/mediacentre/factsheets/fs312/es/index.html. Acceso el 9 de Noviembre de 2012.

4. Ruz-Ramos M, Escolar-Pujolar A, MayoralSánchez E, Corral-San Laureano F, FernándezFERNÁNDEZ I. La diabetes mellitus en España: mortalidad, prevalencia, incidencia, costes económicos y desigualdades. Gac Sanit 2006; 20: $15-24$.

5. Valdés S, Rojo-Martínez G, Soriguer F. Evolución de la prevalencia de la diabetes tipo 2 en población adulta española. Med ClinBarcelona 2007; 129: 716-717.

6. CAPRIO S. Insulin resistance in childhood obesity. J Pediatr Endocr Met 2002; 15: 487-492.

7. Gellar LA, Schrader K, Nansel TR. Healthy eating practices: perceptions, facilitators, and barriers among youth with diabetes. Diabet Educ 2007; 33: 671-679.

8. Kralik D, Koch T, Price K, Howard N. Chronic illness selfmanagement: taking action to create order. J Adv Nurs 2003; 13: 259-267.

9. KENNEDy A, Rogers A, Bower P. Support for self care for patients with chronic disease. BMJ 2007; 335: 968-970.

10. Chen HS, Wu TE, JAP TS, LIN SH, HSiao LC, LiN HD. Improvement of glycaemia control in subjects with type 2 diabetes by self-monitoring of blood glucose: comparison of two management programs adjusting bedtime insulin dosage. Diabetes Obes Metab 2008; 10: 34-40.

11. Gary Tl, Genkinger JM, Guallar E, Peyrot M, BRANCATI FL. Meta-analysis of randomized educational and behavioral interventions in type 2 diabetes. Diabet Educ 2003; 29: 488-501.

12. Lee TI, Yeh YT, Liu CT, Chen PL. Development and evaluation of a patient-oriented education system for diabetes management. Int $\mathbf{J}$ of Med Inform 2007; 76: 655-663.

13. Phillips LS, Ziemer DC, Doyle JP, Barnes CS, Kolm P, Branch WT et al. An endocrinologistsupported intervention aimed at providers improves diabetes management in a primary care site: improving primary care of African Americans with diabetes (IPCAAD) 7. Diabet Care 2005; 28: 2352-2360.
14. Ralston JD, Hirsch IB, Hoath J, Mullen M, ChEAdle A, GoldBerg HI. Web-based collaborative care for type 2 diabetes: a pilot randomized trial. Diabet Care 2009; 32: 234-239.

15. Rayman K, Eluison G. Home alone: the experience of women with type 2 diabetes who are new to intensive control. Health Care Women Int 2004; 25: 900-915.

16. Sun J, Wang Y, Chen X, Chen Y, Feng Y, Zhang X et al. An integrated intervention program to control diabetes in overweight Chinese women and men with type 2 diabetes. Asia Pac J Clin Nutr 2008; 17: 514-524.

17. Tessier DM, Lassmann-Vague VJ. Diabetes and education in the elderly. Diabetes Metab 2007; 33: 75-78.

18. Holmström IM, Rosenqvist U. Misunderstandings about illness and treatment among patients with type 2 diabetes. J Adv Nurs 2005; 49: 146-154.

19. LIPPA KD, KLEIN HA. Portraits of Patient Cognition: How Patients Understand Diabetes SelfCare. Can J Nurs Res 2008; 40: 80-95.

20. LaI WA, LEW-Ting CY, Chie WC. How diabetic patients think about and manage their illness in Taiwan. Diabet Med 2005; 22: 286-292.

21. Sturt J, Hearnshaw H, Barlow J.H, Hainsworth J, WhitLock S. Education for people with type 2diabetes: What do patients want? J Diabet Nurs 2005; 9: 145-150.

22. Аввотт S, GunNell C. Older people's experiences of diabetes care. J Diabet Nurs 2004; 8: $182-84$.

23. AdAms CR. Lessons learned from urban Latinas with type 2 diabetes mellitus. J Transcult Nurs 2003; 14: 255-265.

24. The Joanna Briggs Institute (sitio en Internet). Disponible en: http://www.jbiconnectplus.org/. Acceso el 12 de Enero de 2013.

25. Wellard SJ, RenNie S, King R. Receptions of people with type 2 diabetes about self-management and the efficacy of community based services. Contemp Nurs 2008; 29; 218-226.

26. Hall RF, Joseph DH, Schwartz-Barcott D. Overcoming obstacles to behaviour change in diabetes self-management. Diabet Educ 2003; 29: 303-311.

27. Nunez MA, Yarandi H, Nunez-Smith M. Self-management among patients living with Diabetes in the United States Virgin islands. J Health Care Poor U 2011; 22: 271-283.

28. Barnes L, Moss-Morris R, KaUfusi M. Illness beliefs and adherence in diabetes mellitus: a comparison between Tongan and European patients. NZ Med 2004; 117 (1188): U743U751. 
29. Lin CC, Anderson RM, Hagerty BM, Lee BO. Diabetes self-management experience: a focus group study of Taiwanese patients with type 2 diabetes. J Clin Nurs 2008; 17(5a): 34-42.

30. Wong M, Haswell-Elkins M, Tamwoy E, McDerмотт R, D’Aввs P. Perspectives on clinic attendance, medication and foot-care among people with diabetes in the Torres Strait Islands and Northern Peninsula Area. Aust J Rural Health 2005; 13: 172-177.

31. Song Y, Song HJ, Han HR, Park SY, Nam S, Kim MT. Unmet Needs for Social Support and Effects on Diabetes Self-care Activities in Korean Americans With Type 2 Diabetes. Diabet Educ 2012; 38: 77-85.

32. Ruiz Olabuenaga, Ji. Metodología de la investigación cualitativa. 4 ed. Bilbao: Universidad de Deusto; 2012.

33. Marriner-Tomey A, Alligood MR. Modelos y teorías en enfermería. 6 ed. Madrid: Elsevier Mosby; 2007.

34. Lundberg PC, Thrakul S. Type 2 diabetes: how do Thai Buddhist people with diabetes practise self-management? J Adv Nurs 2012; 68: 550-558.

35. LoHrI-Posey B. Middle-aged Appalachians living with diabetes mellitus: a family affair. Fam Community Health 2006; 29: 214-220.

36. Caban A, Walker EA, Sanchez S, Mera MS. "It Feels Like Home When You Eat Rice and Beans": Perspectives of Urban Latinos Living With Diabetes. Diabetes Spectr 2008; 21: 120127.

37. Fleming E, Carter B, Pettigrew J. The influence of culture on diabetes self-management: perspectives of Gujarati Muslim men who reside in northwest England. J Clin Nurs 2008; 17 (5a): 51-59.

38. Sowattanangoon N, Kotchabhakdi N, Petrie KJ. The influence of Thai culture on diabetes perceptions and management. Diabetes Res Clin Pract 2009; 84: 245-251.

39. Wang Y, Chuang L, Bateman WB. Focus Group Study assessing self-management skills of Chinese Americans with type 2 diabetes mellitus. J Immigr Minor Health 2012; 14: 869874.

40. WeILER DM, CRIST JD. Diabetes Self-management in a Latino Social Environment. Diabet Educ 2009; 35: 285-292.

41. Carter-Edwards L, Skelly AH, Cagle CS, Appel SJ. They care but don't understand: family support of African American women with type 2 diabetes. Diabet Educt 2004; 30: 493501.
42. Burke JA, Earley M, Dixon LD, Wilke A, PuczynsKI S. Patients with diabetes speak: exploring the implications of patients' perspectives for their diabetes appointments. Health Commun 2006; 19: 103-114.

43. Pilkington FB, Daisuki I, Bryant T, Dinca-Panaitescu M, Dinca-Panaitescu S, Raphael D. The experience of living with diabetes for low-income Canadians. Can J Diabetes 2010; 34: 119-126.

44. Jones RA, Utz SW, Williams IC, Hinton I, AlexanDER G, MoOre C et al. Family interactions among African Americans diagnosed with type 2 diabetes. Diabet Educ 2008; 34: 318326.

45. Handley J, Pullon S, Gifford H. Living with type 2 diabetes: Putting the person in the pilots seat. Aust J Adv Nurs 2010; 27: 12-19.

46. Ramsay Wan C, Vo L, BaRnes CS. Conceptualizations of patient empowerment among individuals seeking treatment for diabetes mellitus in a urban, public-sector clinic. Patient Educ Couns 2012; 87: 402-404.

47. Nagelkerk J, Reick K, Meengs L. Perceived barriers and effective strategies to diabetes self-management. J Adv Nurs 2006; 54: 151158.

48. JOHnSON M, Baird W, GoYder E. Understanding issues involved in the transfer of diabetes care to general practice: the patient perspective. Qual Prim Care 2006; 14: 247-252.

49. Oftedal B, Karlsen B, Bru E. Perceived support from healthcare practitioners among adults with type 2 diabetes. J Adv Nurs 2010; 66 (7): 1500-1509.

50. Hornsten A, Lundman B, Selstam EK, Sandstrom H. Patient satisfaction with diabetes care. J Adv Nurs 2005; 51: 609-617.

51. Lawton J, Peel E, Parry O, Araoz G, Douglas M. Lay perceptions of type 2 diabetes in Scotland: bringing health services back in. Soc Sci Med 2005; 60: 1423-1435.

52. Lawton J, Rankin D, Peel E, Douglas M. Patients' perceptions and experiences of transitions in diabetes care: a longitudinal qualitative study. Health Expect 2009; 12: 138-148.

53. Ministerio de Sanidad, Servicios Sociales e Igualdad. Estrategia para el abordaje de la Cronicidad en el Sistema Nacional de Salud. Madrid: Ministerio de Sanidad, Servicios Sociales e Igualdad, 2012.

54. Ministerio de Sanidad, Servicios Sociales e Igualdad. Estrategia en Diabetes del Sistema Nacional de Salud (Actualización). Madrid: Ministerio de Sanidad, Servicios Sociales e Igualdad, 2012. 